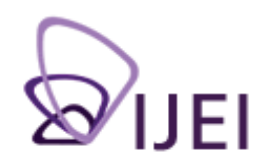

International Journal for Educational Integrity

\title{
Core elements of exemplary academic integrity policy in Australian higher education
}

\author{
Tracey Bretag
}

School of Management, University of South Australia

tracey.bretag@unisa.edu.au

\section{Saadia Mahmud}

School of Management, University of South Australia

saadia.mahmud@unisa.edu.au

\section{Margaret Wallace}

Centre for Educational Development, Innovation \& Recognition, University of Wollongong.

mwallace@uow.edu.au

\section{Ruth Walker}

Learning Development Academic Services Division, University of Wollongong. rwalker@uow.edu.au

\section{Colin James}

Newcastle Law School, University of Newcastle

colin.james@newcastle.edu.au

\section{Margaret Green}

School of Health Sciences, University of South Australia

margaret.green@unisa.edu.au

\section{Julianne East}

Academic Language and Learning Unit, La Trobe University

j.east@latrobe.edu.au

\section{Ursula McGowan}

Centre for Learning and Professional Development, University of Adelaide.

ursula.mcgowan@adelaide.edu.au

\section{Lee Partridge}

Higher Education Development, University of Western Australia

lee.partridge@uwa.edu.au

Keywords: academic integrity, academic integrity policy, higher education

\section{Abstract}

This paper reports on one important aspect of the preliminary findings from the Australian Learning and Teaching Council (ALTC) project, Academic integrity standards: Aligning policy and practice in Australian universities (Bretag et al., 2010) Our project aims to identify approaches to the complex issues of academic integrity, and then to build on these approaches to develop exemplars for adaptation across the higher education sector. Based on analysis of publicly available online academic 
integrity policies at each of the 39 Australian universities, we have identified five core elements of exemplary academic integrity policy. These have been grouped under the headings, Access, Approach, Responsibility, Detail and Support, with no element given priority over another. In this paper we compare the five core elements identified in our research with best practice guidelines recommended by the Higher Education Academy (HEA) in the UK. We conclude that an exemplar policy needs to provide an upfront, consistent message, reiterated throughout the entire policy, which indicates a systemic and sustained commitment to the values of academic integrity and the practices that ensure it. Whereas the HEA created two discrete resources, the key aim and challenge of this project will be to develop exemplars that demonstrate a strong alignment between policy and practice.

\section{Introduction}

How a university defines and explains the role of academic integrity (AI) in its policy will affect the way it is taught and embedded in the curriculum. It therefore follows that policies, procedures, teaching and assessment practices should be interconnected. In the UK, the call to examine consistency in academic integrity came from the Independent Adjudicator for Higher Education who declared that variation in penalties for plagiarism across the higher education sector was indefensible. This led to the development of the project, 'Academic Misconduct Benchmarking Research (AMBeR)' (Tennant, Rowell, \& Duggan, 2007). In the Australian higher education context, the need for consistency in academic integrity was highlighted by the AUQA audit of one university which found inconsistent practice in the application of academic honesty information and testing across the faculties as well as lost opportunities to educate students about academic integrity, and potentially inconsistent application of penalties (AUQA, 2010). Cognisant of the value of consistency, this ALTC project seeks to uncover evidence of this congruence by, amongst other things, analysing university policy in relation to academic integrity.

The project adopts the University of Tasmania statement on academic integrity:

Academic integrity is about mastering the art of scholarship. Scholarship involves researching, understanding and building upon the work of others and requires that you give credit where it is due and acknowledge the contributions of others to your own intellectual efforts. At its core, academic integrity requires honesty. This involves being responsible for ethical scholarship and for knowing what academic dishonesty is and how to avoid it. (University of Tasmania, 2010)

Although students have different experiences of scholarly practice, these core principles of academic integrity apply to all: undergraduate and postgraduate, coursework and higher degree by research students. In addition, it is important to acknowledge that academic integrity is "multi-dimensional and is enabled by all those in the educational enterprise, from students to teachers, librarians, advisors, research colleagues and administrators" (APFEI, 2010).

With the aim of establishing collaboratively devised resources and exemplars that demonstrate an alignment of Al policy with practice, we draw on the work of East (2009), who has provided "a checklist for an aligned approach to implementing academic integrity". East (2009) makes the compelling case that in order to inculcate and foster academic integrity, universities need to align policy, teaching and learning practices, Al decision-making and Al review processes. As Figure 1 illustrates, we maintain that a culture of academic integrity is central to all aspects of policy and practice. This diagram indicates how each of the four aspects identified by East (2009) revolve around and contribute to the fostering of Al culture, and are in turn, informed and transformed in a continuous cycle of reflexivity. 


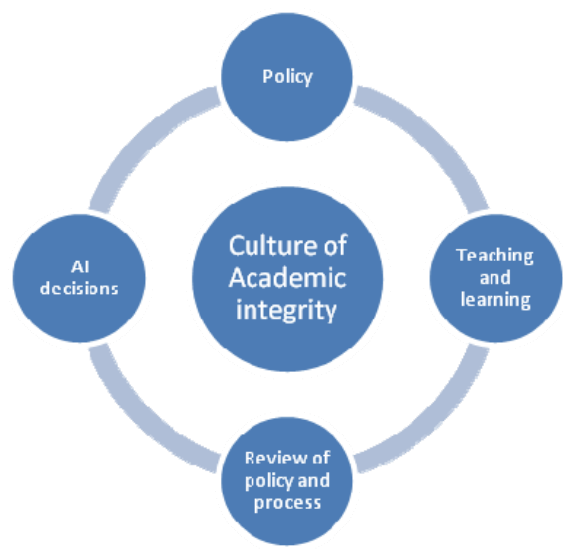

Figure 1: Aligning policy and practice in a culture of academic integrity (extending East, 2009).

This paper responds to the policy aspect in Figure 1 above and reports on the findings from an analysis of Australian universities' academic integrity policies.

\section{Method}

The academic integrity policies of 39 Australian universities were accessed online and analysed during the period of December 2010 to March 2011. Locating the appropriate policy at each of the universities was not a simple task as many universities have multiple, related, overlapping but not always linked or updated policies. The approach taken was to determine the main document related to academic integrity policy and only consider additional documents if embedded links were provided in the main policy document.

The project team members were each allocated a specified number of academic integrity policies to code, with at least two coders responsible for every policy. Preliminary coding was based on the literature, our own experience, as well as a recent doctoral dissertation that examined the plagiarism policies of Australian universities (Grigg, 2010).

A total of 20 preliminary categories were used in the first stage of the analysis. These categories are listed below:

1. Title of the policy.

2. Key terms.

3. Definition of academic integrity: Whether academic integrity or academic honesty are defined in the policy.

4. Related embedded documents.

5. Purpose: Clear statement of the purpose of the policy.

6. Responsibility: Clear statement of responsibility for each stakeholder in the policy.

7. Breach ID: Identification of what constitutes a breach of the policy.

8. Whether the policy uses the terms intent/intention/motivation/knowingly to determine breach behaviour.

9. Tool used to detect plagiarism (e.g. manual process, detection software).

10. Levels: Classification of a breach according to levels/tiers/major or minor.

11. Approach: The spirit of the policy, whether to punish, educate, minimise risk, or develop integrity.

12. Penalty: Outcomes of the policy breach are stated.

13. Mention of the term 'collusion' used in policy or assisting breach. 
14. Whether the policy applies to higher degree by research (HDR) students.

15. Whether the policy mentions retrospective application.

16. Reporting: Who notifies of a breach of policy?

17. Recording: Where is a breach of policy recorded?

18. Confidentiality: Whether the record of breaches is kept confidential and what is the level of access.

19. Ease of access: Ability to find the policy on the university's website using the search function and any of the following terms: academic integrity policy, plagiarism policy, academic honesty policy.

20. Timing: When the policy was last reviewed or approved.

Based on the team's discussion, following individual analysis, an additional two categories were included in the coding during the second stage of analysis. These categories are listed below:

21. Circumstances: Context, mitigating circumstances, factors to consider regarding breach.

22. Enabling implementation: Procedures, resources, modules, training, seminars, and professional development activities to facilitate staff and student awareness and understanding of policy.

The second stage of analysis was conducted on 12 policies that were shortlisted as potential exemplars from the initial 39 academic integrity policies. Each shortlisted policy was rated on a five-point scale ( 1 being the highest and 5 being the lowest) and the overall strengths and weaknesses of the policy were summarised by the reviewers. The rating given to a policy was based on a number of considerations such as the policy having an explicit purpose with introductory material that provided context for the policy; a statement of scholarly values with an institutional commitment to academic integrity; an appropriate level of detail in the section on breach identification including definitions with examples; an outline of responsibilities for all stakeholders, e.g. the university, staff, and students; and detailed procedures and penalties. The policy also needed to be easy to read, well written, concise, with logical headings, and links to resources.

Each exemplar policy was reviewed by two team members independently and the ratings were compared and discussed as a team. The policies that received a rating of 1 by at least one reviewer and had converging reviews between the two reviewers and the final review by the project team leader and project manager, were considered as exemplar academic integrity policies. The ratings by the reviewers were within one rating point for all the policies with one exception where the rating was apart by two rating points.

\section{Results and discussion}

As a consequence of the second stage of analysis, six academic integrity policies were identified as potential exemplars from the 12 shortlisted policies. The following discussion of the elements of an academic integrity policy exemplar is drawn primarily from these shortlisted six policies. As the project progresses, resources will be developed based on these exemplar academic integrity policies. The five elements of exemplary academic integrity policy are detailed below:

1. Access: The policy is easy to locate, easy to read, well written, clear and concise. The policy uses comprehensible language, logical headings, provides links to relevant resources and the entire policy is downloadable as an easy to print and read document. This element is given priority in this list, because no matter how comprehensive or well developed a policy, if it is not accessible and 
understandable to both staff and students, it would be unlikely to be implemented effectively.

2. Approach: Academic integrity is viewed as an educative process and appears in the introductory material to provide a context for the policy. There is a clear statement of purpose and values with a genuine and coherent institutional commitment to academic integrity through all aspects of the policy. This aspect needs to be one that necessarily runs through all other elements of the policy. An exemplary approach does not begin and end with an upfront statement of intent, but influences both the language and the substance of the entire policy.

3. Responsibility: The policy has a clear outline of responsibilities for all relevant stakeholders, including university senior management, academic and professional staff, and students. The approach identified by Bertram Gallant and Kalichman (2011) is an example of how a systems approach could be embedded in an exemplary policy. This incorporates responsibility for academic integrity at the individual, organisation, education system and social levels.

4. Detail: The policy provides a detailed description of a range of academic integrity breaches and explains those breaches using easy to understand classifications or levels of severity. Details of how breaches are identified (such as through the use of text-matching software) are provided. Processes are detailed with a clear list of objective outcomes, and the contextual factors relevant to academic integrity breach decisions are outlined. Extensive but not excessive detail is provided in relation to reporting, recording, confidentiality and the appeals process. Exemplary policy incorporates simple flow charts to demonstrate how the policy is enacted in practice.

5. Support: Systems are in place to enable implementation of the academic integrity policy including procedures, resources, modules, training, seminars, and professional development activities to facilitate staff and student awareness and understanding of policy. For example, proactive measures to educate students about academic writing and referencing conventions as well as practical strategies to prevent breaches of academic integrity. Enabling strategies enact the policy. Without long-term, sustainable and practical support resources, a policy will not be enacted, no matter how well it is articulated.

Figure 2 below depicts the five elements of academic integrity policy: Access, Approach, Responsibility, Detail and Support. In keeping with our theoretical and philosophical stance (based on the work of East, 2009), we maintain that an overarching commitment to a culture of academic integrity lies at the heart of an exemplar academic integrity policy because without this commitment the five elements do not work coherently and consistently together.

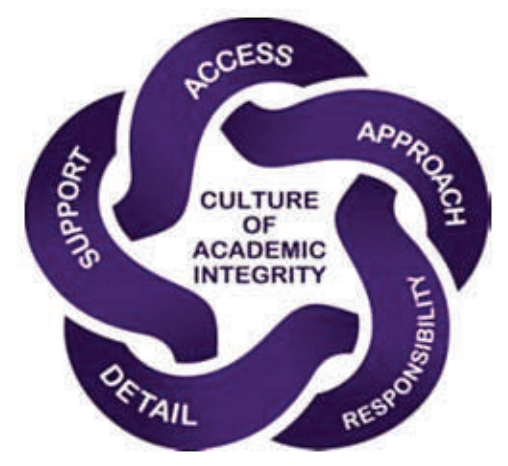

Figure 2: Core elements of exemplar academic integrity policy. 
We have chosen 'Solomon's Seal Knot' (also known as a Cinquefoil or Pentafoil knot) to represent the elements of exemplar policy for three reasons: the first is that this knot demonstrates that the five elements are all connected, as part of just one strand. The elements do not exist as separate and discrete aspects, and nor is any one element privileged over another. Second, one could imagine that if the knot was pulled together, it would represent great strength - it would, in essence, become a culture of academic integrity, just as the combined force of all the elements work together to strengthen the effectiveness of policy on practices within an institution. The third reason for our choice of this representation is the historical association of wisdom and prudent governance with Solomon's Seal (Moore, 1999). In presenting our vision for exemplar policy we are advocating a stance that goes beyond traditional notions of knowledge. We maintain that wisdom is needed to understand both the centrality of academic integrity to higher education, and the importance of strong governance based on clear ethical principles.

The UK HEA (HEA, 2011a) has provided recommendations for good practice in academic integrity policy which correspond to the five core elements identified in our research. Six of the 12 recommendations directly correspond to what we have categorised as 'Detail'. The HEA's recommendations are complemented by a separate resource document, which provides support for enacting the policy. In combination, these two documents provide a useful anchor to our own research and for the resources which will eventually be developed as part of this ALTC project.

Table 1 below compares the focus of the recommendations by the HEA (2011a) for academic integrity policy, with the core elements of academic integrity policy identified by our research.

Table 1:

Comparison of core elements of academic integrity policy with HEA recommendations

\begin{tabular}{|c|c|}
\hline $\begin{array}{l}\text { Core elements of } \\
\text { academic integrity } \\
\text { policy identified in our } \\
\text { research }\end{array}$ & $\begin{array}{l}\text { Focus of Higher Education Academy } \\
\text { recommendations for academic integrity policy }\end{array}$ \\
\hline Access & - Establish a central web area on the institutional website. \\
\hline Approach & $\begin{array}{l}\text { - Establish cross-institutional group or committee. } \\
\text { - Include statements about the importance of academic scholarship } \\
\text { and honesty in policy and related guidance for unacceptable } \\
\text { academic practice, where the principles and values for academic } \\
\text { integrity and academic practice are considered. }\end{array}$ \\
\hline Responsibility & $\begin{array}{l}\text { - Establish cross-institutional group or committee. } \\
\text { - Make explicit the responsibilities of the institution, staff and students. }\end{array}$ \\
\hline Detail & $\begin{array}{l}\text { Make explicit the strategies used to identify possible instances of } \\
\text { unacceptable academic practice. } \\
\text { - Develop documentation for policy and procedures that are well } \\
\text { structured, and easy to understand, use and follow. } \\
\text { - Carefully consider terminology, definitions and associated examples. } \\
\text { - Provide clear and detailed procedures for reporting and managing } \\
\text { cases of unacceptable academic practice. } \\
\text { - Establish a set of available penalties with associated guidance so } \\
\text { that staff can determine appropriate penalties that are fair and } \\
\text { proportionate. } \\
\text { Establish a centralised system to record and monitor cases of } \\
\text { unacceptable academic practice. }\end{array}$ \\
\hline Support & $\begin{array}{l}\text { - Ensure a variety of strategies and mechanisms to inform and } \\
\text { educate students. } \\
\text { - Develop strategies for staff engagement and development. }\end{array}$ \\
\hline
\end{tabular}




\section{Access}

Consistent with our own analysis, the HEA makes the case for easily accessed information as a high priority (in the top 3 out of 12). Access might appear to be a mere technicality but it is crucial to make effective the other elements, and so needs to be valued as much as the others. Without simple and easy access for all stakeholders, any policy may as well not exist. Our research showed that 6 of the 39 universities $(15 \%)$ had policies which were difficult to locate, in terms of easily finding the relevant information. Our research did not include a more nuanced analysis of Access as we have defined it here.

\section{Approach}

Our analysis indicated that Approach was crucial to all other elements of the policy in that it influenced and directed both the language and the substance of the entire policy. Some universities attempted to preface their policies with an upfront statement of purpose, but in then providing extensive detail about 'penalties' and 'misconduct', lost the original vision. As we have reported elsewhere, $28 \%$ of policies had approaches which we analysed as having a mix of both educative and punitive elements which may have ramifications for the way that academic integrity is perceived by students and staff (Bretag et al., 2011). While we agree with the HEA's recommendation for 'statements about the importance of academic scholarship and honesty', we maintain that simple statements are not enough. An exemplar policy needs to provide an upfront, consistent message reiterated throughout the entire policy which indicates a systemic and sustained commitment to the values of academic integrity and the practices that ensure it.

\section{Responsibility}

Our research indicates that multiple stakeholder Responsibility is a core element of exemplar policy. In line with Bertram Gallant and Kalichman (2011), we consider that this responsibility extends beyond the institution and into the educational system and social spheres. An exemplar policy will remind all stakeholders that integrity is not confined to the academy, but in fact has ramifications for personal and professional practice. Those outside the university (e.g. parents, friends, colleagues, employers) also have a role to play. Our research demonstrated that 8 of the 39 universities $(21 \%)$ placed all of the responsibility for academic integrity in the hands of students (Bretag et al., 2011). Only one university specifically stated that 'everyone' is responsible.

\section{Detail}

Half of the UK HEA (HEA, 2011a) recommendations correspond to the core element we have identified as Detail. While we agree that extensive but not excessive detail is vital to an academic integrity exemplar policy, our analysis has not given this the same weighting. During the coding process, it was apparent that many Australian universities had substituted detail for comprehensible and coherent educational content centred on a commitment to academic integrity for all stakeholders. It seemed that many universities had invested effort in developing a student-targeted, detailed policy which provided information about what would happen if a student breached the policy, but without a central, clearly articulated vision which explained why this detail was necessary. We agree that detail is very important, but as just one element of five equally important aspects, rather than the key ingredient.

One point worth noting is the lack of discussion about issues of confidentiality in the HEA's recommendation relating to record keeping of academic integrity breaches. Our research has shown that 22 (or $56 \%$ ) of the 39 Australian universities did not refer to confidentiality in their policies. Only $15(38 \%)$ explicitly stated that records would be kept confidential. This is an important issue as anecdotal evidence suggests that both staff and students are apprehensive that any reporting of an Al breach will be 'held against' the student by future lecturers or administrators. Staff may be 
reluctant to report a possible breach, and students may feel justifiably anxious if they cannot be assured that Al investigations are confidential.

\section{Support}

Support is crucial for the enactment of exemplar policy. In keeping with our systems approach, we have not delineated the support offered to staff from that provided to students, although of course we do recognise that these distinct stakeholder groups require specific and targeted support, unique to each group. In fact, our approach goes one step further than the simple recommendation of the HEA to "ensure that policy and procedures are consistently followed" (2011a, p. 12). In our opinion, the types of professional development activities likely to engage staff go well beyond adherence to policy, and in addition, encourage staff to reflect on fundamental issues relating to academic values, ethical scholarship and their own research practices. This project, however, maintains the view that support needs to be embedded in the policy and be central to it rather than separate. Whereas the HEA created two discrete resources, Policy Works (HEA, 2011a) and Supporting Academic Integrity (HEA, $2011 \mathrm{~b}$ ), the key aim and challenge of this project will be to develop exemplars that demonstrate a strong alignment between policy and practice.

\section{Conclusion}

In this paper we have identified five core elements of exemplary academic integrity policy, based on preliminary analysis of the publicly available online academic integrity policies at each of the 39 Australian universities. These have been categorised as Access, Approach, Responsibility, Detail and Support, and diagrammatically represented as a five stranded 'seal knot' to emphasise the interrelated and non-hierarchical nature of the elements. We have also juxtaposed the identified elements with best practice guidelines recommended by the HEA in the UK to determine any gaps or potential areas for development. In company with the HEA, the intention of this project is not to provide a standardised model for approaches to academic integrity. Rather we hope to develop a more sophisticated approach that goes beyond a policy-level mechanism presuming to cut across the sector, at intra- and inter-university levels, and across courses and disciplines. With Sutherland-Smith (2010), and other leaders in the field of academic integrity (Macdonald \& Carroll, 2006; Bertram Gallant, 2011), our research points to the need for far-reaching reform in higher education - one that works from both the ground up and the top down to instigate ethical scholarship and practice at all levels.

We are confident that the opportunity provided by examination and discussion of policy and practice across the sector will lead to individual institutional reflection of how their academic integrity policies might be enhanced and better implemented. The core elements of exemplar academic integrity policy identified in this paper aim to begin a dialogue which will encourage a more nuanced and effective understanding of academic integrity in the Australian higher education context.

\section{References}

Asia Pacific Forum on Educational Integrity (APFEI) (2010). What is educational integrity? Retrieved June 25, 2010 from http://apfei.edu.au/tiki-index.php

Australian Universities Quality Agency (AUQA) (2010). Audits: Universities. Retrieved February 28, 2010 from http://www.auqa.edu.au/qualityaudit/universities/

Bertram Gallant, T. (Ed.). (2011). Creating the ethical academy: A systems approach to understanding misconduct and empowering change in higher education. New York: Routledge.

Betram Gallant, T., \& Kalichman, M. (2011). Academic ethics: A systems approach to understanding misconduct and empowering change in the academy. In T. Betram Gallant (Ed.), Creating the ethical academy: A systems approach to 
understanding misconduct and empowering change in higher education (pp. 27 -44). New York: Routledge.

Bretag, T., Mahmud, S., East, J., Green, M., James, C., McGowan, U., Partridge, L., Wallace, M., \& Walker, R. (2011). Academic integrity standards: A preliminary analysis of the academic integrity policies at Australian universities. Refereed paper accepted at the Australian Quality Forum, 29 June-1 July, Melbourne, Australia. Retrieved http://www.auqa.edu.au/files/auqf/paper/paper_h20.pdf

Bretag, T., Walker, R., Green, M., Wallace, M., East, J., James, C., McGowan, U., \& Partridge, L. (2010). Academic integrity standards: Aligning policy and practice in Australian universities, successful proposal to the Australian Learning and Teaching Council, Priority Projects, Round 2.

East, J. (2009). Aligning policy and practice: An approach to integrating academic integrity. Journal of Academic Language and Learning, 3(1), A38-A51. Retrieved http://journal.aall.org.au/index.php/jall/article/viewFile/66/62

Grigg, G. (2010). Plagiarism in higher education: Confronting the policy dilemma, Unpublished PhD University of Melbourne, Melbourne, Australia. Retrieved http://repository.unimelb.edu.au/10187/8971

Higher Education Academy JISC Academic Integrity Service (2011a). Policy works: recommendations for reviewing policy to manage unacceptable academic practice in higher education. Retrieved May 24, 2011, from http:// www.heacademy.ac.uk/resources/detail/academicintegrity/policy_works

Higher Education Academy JISC Academic Integrity Service (2011b). Supporting academic integrity: Approaches and resources for higher education. Retrieved May 24, 2011 from http://www.heacademy.

ac.uk/assets/documents/academicintegrity/SupportingAcademicIntegrity_v2.pdf

Macdonald, R., \& Carroll, J. (2006). Plagiarism - a complex issue requiring a holistic institutional approach. Assessment \& Evaluation in Higher Education, 31(3), 233-245.

Moore, B. (Ed.). (1999). Australian Oxford Dictionary. Melbourne, Australia: Oxford University Press.

Sutherland-Smith, W. (2010). Retribution, deterrence and reform: The dilemmas of plagiarism management in universities. Journal of Higher Education Policy and Management, 32(1), 5-16. http://dx.doi.org/10.1080/13600800903440519

Tennant, P., Rowell, G., \& Duggan, F. (2007). AMBeR Project. Joint Information Systems Committee (JISC). Retrieved March 20, 2008, from http://www.jisc.ac.uk/news/stories/2008/06amber.aspx

University of Tasmania (2010). Academic integrity. Retrieved June 25, 2010, from http://www.academicintegrity.utas.edu.au.

\section{About the authors}

Tracey Bretag, BA (Hons), MA, EdD (by research), Senior Lecturer and Academic Integrity Officer, School of Management, University of South Australia. Bretag's interest in the topic began with her own doctoral work, 'Implementing plagiarism policy in the internationalised university' (Bretag, 2005). Bretag is the founding editor of the International Journal for Educational Integrity, a member of the advisory Board of the International Center for Academic Integrity, and Co-Chair (with Ruth Walker) of the Asia Pacific Forum on Educational Integrity.

Saadia Mahmud, MBA, MPhil (Management), Grad Dip Fin Plan, PhD, Research Associate, School of Management, University of South Australia. After a decade of working in banking and finance, Mahmud joined UniSA in 2001. Mahmud's doctoral thesis entitled 'Role of self-organisation in the handling of adaptive challenges by enterprises' (Carapiet, 2006) found that open and honest communication and trust were related to the ability to self-organise. The vital role of open and honest communication, and trust in organisations is a recurrent theme in her research, including her work with Bretag on plagiarism $(2007,2009)$. 
Margaret Wallace, BA, GradDipEd (Nursing), PostgradDipNursing (Midwifery), MEd, $\mathrm{PhD}$, is an Associate Professor, Curriculum Development and Review, Academic Development Unit (secondment) at the University of Wollongong. Wallace Co-Chaired the 4th Asia Pacific Conference on Educational Integrity in 2009, and co-convened the Academic Integrity Symposium at UOW in 2008. She has been active in promoting academic integrity in the field of Health and Behavioural Science.

Ruth Walker, BA (Hons), PhD, Learning Development, University of Wollongong. An Early Career Researcher, Walker Co-Chaired the 4th Asia Pacific Conference on Educational Integrity at UOW in 2009, is currently Co-Chair (with Tracey Bretag) of APFEl and the Guest Editor for the IJEI Vol 6(2) 2010. Walker was awarded an ALTC Outstanding Contribution to Student Learning Citation 2008 for "sustained work to collaboratively integrate contextualised academic integrity into curricula". Walker's research interests are in affective theories of plagiarism and the impact of digital media to research and writing practices.

Colin James, BA, BJuris, LLB, MPhil, PhD, GCertPTT, Newcastle Law School, University of Newcastle. James is a lawyer/researcher, a senior academic, a member of the University of Newcastle Research Ethics Committee and a member of several management committees and community reference groups in the community sector. $\mathrm{He}$ has a strong interest in ethical practice, clinical legal supervision and professional responsibility. He has been a member of APFEI since 2005 and is responsible for drafting its constitution. His research interests include the intersections of mindfulness, emotional intelligence and professional development.

Margaret Green, Dip Physio, MAppSc (Physio), Lecturer in Health Science, Academic Integrity Officer, University of South Australia. Green has been a core member of APFEl since 2003, and in 2007 Co-Chaired (with Bretag) the 3rd AsiaPacific Conference on Educational Integrity: Creating a Culture of Integrity, at the University of South Australia.

Julianne East, BA, DipEd, PostGrad DipEd, MEd, PhD, La Trobe University; Chair, La Trobe University Turnitin Users Group; Resource Developer, Academic Integrity website La Trobe University. East has been an integral member of APFEI since it was formed in 2003, and has been instrumental in promoting academic integrity at La Trobe University. East's (2009) framework for aligning academic integrity policy and practice provides the theoretical foundation for the ALTC project.

Ursula McGowan, BA (Hons), Dip Ed, Grad Dip Ed, MA, Deputy Director, Centre for Learning and Professional Development, University of Adelaide. McGowan has been researching and writing about academic integrity since the 1990s. Her professional focus on student learning.

Lee Partridge, BSc (Hons), Grad Dip Ed, EdD, Academic Developer in the University of Western Australia's Centre for the Advancement of Teaching and Learning. She has a particular interest in the areas of student experience and academic integrity and has played an integral part in the UWA response to developing students' sense of ethical scholarship. She co-convened the 5th Asia Pacific Conference on Educational Integrity held at UWA in September 2011.

An earlier version of this paper was originally published in the refereed proceedings of the $5^{\text {th }}$ Asia Pacific Conference on Educational Integrity: Culture and values. The University of Western Australia, 26-28 September: http://www.apcei.catl.uwa.edu.au/ procs 